\title{
Renal function and hyperfiltration capacity in lead smelter workers with high bone lead
}

Harry Roels, Robert Lauwerys, Jozef Konings, Jean-Pierre Buchet, Alfred Bernard, Stuart Green, David Bradley, Wyn Morgan, David Chettle

\begin{abstract}
Objective-The study was undertaken to assess whether the changes in urinary excretion of eicosanoids (a decrease of 6keto-PGF Ia $_{1 a}$ and PGF $_{2}$ and an increase of thromboxane) previously found in lead $(\mathrm{Pb})$ exposed workers may decrease the renal haemodynamic response to an acute oral protein load.
\end{abstract}

Methods-The renal haemodynamic response was estimated by determining the capacity of the kidney to increase the glomerular filtration rate (in terms of creatinine clearance) after an acute consumption of cooked red meat $(400 \mathrm{~g})$. A cross sectional study was carried out in 76 male $\mathrm{Pb}$ workers (age range 30 to 60 years) and 68 controls matched for age, sex, socioeconomic state, general environment (residence), and workshift characteristics.

Results-The $\mathrm{Pb}$ workers had been exposed to lead on average for 18 (range 6-36) years and showed a threefold higher body burden of $\mathrm{Pb}$ than the controls as estimated by in vivo measurements of tibial $\mathbf{P b}$ concentration (Pb-T) (geometric mean $66 v 21 \mu \mathrm{g} \mathrm{Pb/g}$ bone mineral). The geometric mean concentrations of $\mathrm{Pb}$ in blood $(\mathrm{Pb}-\mathrm{B})$ and $\mathrm{Pb}$ in urine $(\mathrm{Pb}-$ $U)$ were also significantly higher in the Pb group (Pb-B: $430 v 141 \mu$ g Pb/l; Pb-U: $40 v 7.5 \mu \mathrm{g} \mathrm{Pb} / g$ creatinine). These conditions of chronic exposure to $\mathrm{Pb}$ did not entail any significant changes in the concentration of blood borne and urinary markers of nephrotoxicity, such as urinary low and high molecular weight plasma derived proteins $\left(\beta_{2}\right.$-microglobulin, retinol binding protein, albumin, transferrin), urinary activities of $\mathrm{N}$ acetyl- $\beta$-D-glucosaminidase and kallikrein, and serum concentrations of creatinine, $\beta_{2}$-microglobulin, urea, and uric acid. All participants also had normal baseline creatinine clearances $(>80$ $\mathrm{ml} / \mathrm{min} / 1.73 \mathrm{~m}^{2}$ ) amounting on average to 115.5 in the controls $v 121.3 \mathrm{ml} / \mathrm{min} / 1.73$ $\mathbf{m}^{2}$ in the $\mathbf{P b}$ group. Both control and $\mathbf{P b}$ exposed workers showed a significant increment in creatinine clearance (on average $15 \%$ ) after oral protein load suggesting that the previously found changes in secretion of urinary eicosanoids apparently has no deleterious effect on renal haemodynamics in the examined Pb workers.

Conclusion-The finding that both base- line and stimulated creatinine clearance rates were not only significantly higher in the $\mathbf{P b}$ workers but also positively correlated with $\mathrm{Pb}-\mathrm{T}$, suggests that moderate exposure to $\mathbf{P b}$ may be associated with a slight hyperfiltration state, which has been found to attenuate the age related decline in baseline creatinine clearance by a factor of two. Although the relevance of this effect for the worker's health is unknown, it can be concluded that adverse renal changes are unlikely to occur in most adult male $\mathrm{Pb}$ workers when their blood $\mathbf{P b}$ concentration is regularly kept below $700 \mu \mathrm{gb} / \mathrm{l}$. One should, however, be cautious in extrapolating this conclusion to the general population because of pre-employment screening of the $\mathrm{Pb}$ workers for the absence of renal risk factors.

(Occup Environ Med 1994;51:505-512)

Longstanding occupational exposure to lead $\mathrm{Pb})$ may cause chronic nephrotoxic effects consisting mainly in a decline of the glomerular filtration rate possibly leading to end stage renal insufficiency. ${ }^{1-4}$ The intensity of exposure to $\mathrm{Pb}$ without adverse effects on the kidney is still uncertain because incipient $\mathrm{Pb}$ nephropathy is difficult to diagnose. Most cross sectional studies conducted on $\mathrm{Pb}$ exposed workers have rarely reported renal changes among subjects whose lead in blood (Pb-B) did not regularly exceed $600 \mu \mathrm{g} / 1$ (see Gennart et $a l^{5}$ for review). These findings were recently confirmed by Cárdenas et al ${ }^{6}$ who applied a battery of more than 20 potential markers of renal changes to a cohort of 50 workers moderately exposed to $\mathrm{Pb}(\mathrm{Pb}-\mathrm{B}$ ranging from 360 to $650 \mu \mathrm{g} / \mathrm{l}$; mean duration of exposure 14 years). In this last study, however, changes in the urinary excretion of eicosanoids (decreased urinary excretion of 6keto-PGF ${ }_{1 \alpha}$ and PGE $_{2}$ - two vasodilatorsand an enhanced excretion of thromboxane-a vasoconstrictor) were found to be associated with $\mathrm{Pb}-\mathrm{B}$ or zinc-protoporphyrin concentration in blood (ZPP-B). As the changes in the urinary excretion of eicosanoids likely reflect a disturbance of their synthesis in the kidney, these results raise the suggestion that moderate exposure to $\mathrm{Pb}$ might influence renal haemodynamics. The present study was undertaken to test this hypothesis.

It is known that acute protein consumption 
increases renal perfusion in normal humans leading to a transient hyperfiltration (socalled renal functional reserve). ${ }^{7}$ The mechanism of the increased glomerular filtration rate after an acute protein load has not yet been completely established, but renal production of vasodilatory prostanoids is suspected of playing a part. ${ }^{8-10}$ Therefore we have assessed whether in adult male workers moderately exposed to $\mathrm{Pb}$, the haemodynamic response of the kidney to an oral protein load was different from that found in age matched control subjects. Because examination of the workers had to be carried out in the medical department of a factory, short term measurement of creatinine clearance (Ccr) was considered to be the most practical method for assessing the change in the glomerular filtration rate after acute protein consumption (haemodynamic response $=$ peak $(\mathrm{P})$ Ccr-baseline $(\mathrm{B}) \mathrm{Ccr}$ ). In the context of chronic $\mathrm{Pb}$ exposure current $\mathrm{Pb}-\mathrm{B}$ hardly provides adequate information on the cumulative dose of $\mathrm{Pb}$ to the kidneys. Therefore, the $\mathrm{Pb}$ body burden of the exposed and control workers was assessed by measuring the concentration of $\mathrm{Pb}$ in the tibia $(\mathrm{Pb}-\mathrm{T})$ by $x$ ray fluorescence.

\section{Subjects and methods}

STUDY POPULATION

Workers exposed to $\mathrm{Pb}$ and controls (age range 30 to 60 years) were recruited from the male workforce of a large $\mathrm{Pb}$ smelter in Belgium. Exposed workers had to be occupationally exposed to $\mathrm{Pb}$ for at least five years and their historical $\mathrm{Pb}-\mathrm{B}$ should regularly exceed $300 \mu \mathrm{g} \mathrm{Pb} / 1$. Control workers were selected from the same plant but should never have been directly occupationally exposed to $\mathrm{Pb}$. Current or past $\mathrm{Pb}$ exposure of some control workers might be higher within this plant, however, than that prevailing in the general environment. Also, the occupational history of eligible participants should not show any excessive exposure to cadmium (Cd), mercury $(\mathrm{Hg})$, or other known nephrotoxins. Furthermore, their medical history should not disclose previous EDTA chelation treatment, analgesic misuse, chronic medication for gout, or any other pathological condition (for example, diabetes, past or present renal disease) that might interfere with renal function, except hypertension (subjects on angiotensin converting enzyme inhibitor treatment were excluded).

In view of the possible intricate relation between hypertension, $\mathrm{Pb}$ exposure, and renal function we deliberately included in the study a certain number of $\mathrm{Pb}$ workers with a history of hypertension. Care was taken to ensure that both control and $\mathrm{Pb}$ exposed groups were age matched and had similar socioeconomic state (education, salary), general environment (place of residence), and workshift characteristics. The final database was composed of 68 control and $76 \mathrm{~Pb}$ exposed workers, who may be subdivided into normotensive and hypertensive subgroups. Subjects whose diastolic blood pressure over the past four years never exceeded $90 \mathrm{~mm} \mathrm{Hg}$ were classified as normotensive ( 55 controls and $47 \mathrm{~Pb}$ exposed workers), whereas hypertension was defined as having a diastolic pressure in excess of $90 \mathrm{~mm}$ $\mathrm{Hg}$ or as being on antihypertensive treatment regardless of the measured blood pressure (13 controls and $29 \mathrm{~Pb}$ exposed workers). Five control and six $\mathrm{Pb}$ exposed workers took antihypertensive drugs mainly consisting of $\beta$ blockers.

\section{METHODS}

Protocol for creatinine clearance and protein induced hyperfiltration measurements

The subjects fasted from 2200 the day before the investigation. In each subject, the Ccr was determined before and after a single oral protein load according to a protocol adapted from Bosch et al. ${ }^{11}$ At 0830 the subject drank $400 \mathrm{ml}$ of water and then $200 \mathrm{ml}$ of water every 30 minutes until 1500; timed urine specimens were collected during three periods: from 0900 to 1100 (baseline Ccr) and after protein load, from 1200 to 1330 , and from 1330 to 1500 (first and second period of challenged (Cr); a blood sample was taken in the middle of each of these three periods for the determination of serum creatinine; $400 \mathrm{~g}$ of cooked meat (lean beef) was eaten between 1115 and 1145 . For each subject the higher of the two postmeat $\mathrm{Ccr}$ values was called the peak Ccr, which may occur in either the first or the second period. This protocol was carried out in the medical department of the plant under the supervision of the same investigator. The subjects remained seated and refrained from smoking during the period of examination.

\section{Blood and urine samples}

The collection, handling, and storage of the blood samples and the spot urine samples (about $100 \mathrm{ml}$ urine obtained just before 0900) were carried out exactly as described previously. ${ }^{12}$ The morning blood sample (at 1000) served also to determine the packed cell volume and the concentrations of $Z P P$ in blood (ZPP-B), Cd in blood (Cb-B) and Pb-B, and to obtain serum. An aliquot of $5 \mathrm{ml}$ spot urine was frozen as soon as possible at $-20^{\circ} \mathrm{C}$ for the assay of kallikrein activity; another aliquot $(4 \mathrm{ml})$ was immediately buffered (for analysis of low and high molecular weight proteins), and the remainder of the spot urine was utilised to determine $\mathrm{Cd}-\mathrm{U}, \mathrm{Hg}-\mathrm{U}, \mathrm{Pb}-\mathrm{U}$, $\delta$-aminolevulinic acid ( $\delta$-ALA), and the activity of $\mathrm{N}$-acetyl- $\beta$-D-glucosaminidase (NAG-U).

\section{Biological analyses}

Creatinine concentrations in serum and urine were measured with a Technicon RA1000 (Tarrytown, USA). The concentrations of $\beta_{2}$ microglobulin in urine and serum, and the urinary concentrations of retinol binding protein (RBP), albumin, and transferrin were determined by assays relying on latex particle agglutination. ${ }^{13}$ The determinations of $\mathrm{Cd}-\mathrm{B}$ and $\mathrm{Pb}-\mathrm{B}$ and $\mathrm{Cd}-\mathrm{U}$ were carried out with $\mathrm{a}$ Perkin-Elmer Zeeman 3030 atomic absorption spectrometer. More precise information 
on the analytical procedures for these analyses and the implementation of internal and external quality control programmes have been described elsewhere. ${ }^{14}$ The $\mathrm{Pb}-\mathrm{U}$ was determined according to the APDC/MIBK extraction method of Zinterhofer et al ${ }^{15}$ modified for flameless atomic absorption analysis with a Perkin-Elmer 5000 spectrometer equipped with a deuterium background corrector. Total $\mathrm{Hg}-\mathrm{U}$ was analysed with an automated cold vapour atomic absorption technique ${ }^{16}$ involving $\mathrm{SnCl}_{2}$ for generating elemental $\mathrm{Hg}$. The procedure for the internal quality control of the $\mathrm{Pb}-\mathrm{U}$ analysis has been detailed previously, ${ }^{17}$ and both internal and external quality control programmes were implemented for the analysis of $\mathrm{Hg}-\mathrm{U} \cdot{ }^{17-19}$ The specific measures taken to minimise contamination at the stages of sampling and the analytical procedures have been extensively detailed elsewhere. ${ }^{20}$ Urinary $\delta$-ALA was quantified by the method of Lauwerys et $a l^{21}$ and urinary NAG activity (NAG-U) was assayed fluorimetrically. ${ }^{22}$ Urinary kallikrein activity was determined by a colorimetric method with the chromogenic tripeptide S-2266 (Kabi Vitrum; Stockholm, Sweden). ${ }^{23}$ ZPP-B was measured with a haematofluorimeter (Aviv Associates, Lakewood, NJ, USA). The serum $\gamma$-glutamyl transpeptidase ( $\gamma$-GT) activity and the activity of urea and uric acid in serum were measured by automated techniques with the analysis system ABA-100 (Abbott Laboratories, Irving TX, USA).

\section{In vivo measurement of $\mathrm{Pb}$ in the tibia}

The concentration of tibial $\mathrm{Pb}(\mathrm{Pb}-\mathrm{T})$ was determined with the $\mathrm{K}$ shell $x$ ray fluorescence (XRF) approach developed by the group at Birmingham University and the Queen Elizabeth Medical Centre, UK. ${ }^{24-26}$ The individual measurement precision attained for the examined cohort (control and exposed workers) ranged from \pm 4.9 to $\pm 14.2 \mu \mathrm{g} \mathrm{Pb} / \mathrm{g}$ bone mineral at one standard deviation (SD) with a mean SD of $\pm 7 \cdot 6(n=131)$; hence the practical detection threshold (2 SD) of the measurement system was $15 \cdot 2 \mu \mathrm{g} \mathrm{Pb} / \mathrm{g}$ bone mineral. The measurements were carried out on control and $\mathrm{Pb}$ exposed workers who were scheduled every 30 minutes in an alternating order of sequence. Informed written consent was requested for this measurement from control and $\mathrm{Pb}$ exposed workers of whom 61 and 70 respectively, agreed to participate. The study protocol was approved by the medical ethics committee of the Catholic University of Louvain.

\section{Data processing and statistical analysis}

The creatinine clearances were normalised for $1.73 \mathrm{~m}^{2}$ of body surface area. The difference in $\mathrm{Ccr}$ value $(\Delta \mathrm{Ccr}=$ haemodynamic response $)$ between the postmeat PCcr and the BCcr was calculated; the relative increase of $\mathrm{Ccr}$ was expressed in $\%$ of $\mathrm{BCcr}$ or as the ratio $\mathrm{PC}$ cr/BCcr.

The intrinsic uncertainty associated with the present in vivo tibial $\mathrm{Pb}$ measurement system did not allow precise determinations below $15 \cdot 2 \mu \mathrm{g} \mathrm{Pb} / \mathrm{g}$ bone mineral (see earlier); therefore the $\mathrm{Pb}-\mathrm{T}$ results recorded less than this value in 13 control workers (between -9.2 and $14.7 \mu \mathrm{g} \mathrm{Pb} / \mathrm{g}$ bone mineral) were replaced for statistical calculations by the variance weighted mean $(\overline{\mathrm{Pb}-\mathrm{T}})$ of these 13 values, being $5.4 \mu \mathrm{g} \mathrm{Pb} / \mathrm{g}$ bone mineral ( $\overline{\mathrm{Pb}-\mathrm{T}}=$ $\left.\sum_{\mathrm{i}=1}^{n}\left(\mathrm{~Pb}-\mathrm{T}_{\mathrm{i}}\right) / \sigma_{\mathrm{i}}{ }^{2}\right) / \sum_{\mathrm{i}=1}^{n}\left(1 / \sigma_{\mathrm{i}}\right)^{2}$; where $\mathrm{Pb}-\mathrm{T}_{\mathrm{i}}=$ individual tibia $\mathrm{Pb}$ concentration, $\sigma_{\mathrm{i}}=$ uncertainty associated with $\mathrm{Pb}-\mathrm{T}_{\mathrm{i}}, \mathrm{n}=13$ ).

The Statistical Analysis System (SAS Institute, Cary, NC, USA) for database management and statistical analysis was used. The blood, serum, urine (except urinary flow rate), and $\mathrm{Pb}-\mathrm{T}$ measurements, and also the $\mathrm{Ccr}$ values showed skewed distributions; they were normalised by logarithmic transformation before application of parametric statistics (Student's unpaired $t$ test, paired $t$ test, Yates' $\chi^{2}$ test, analysis of variance and Duncan's multiple range test between subgroups, and simple regression analysis). Multivariate regression analyses were performed to identify $\mathrm{Pb}$ exposure variables (predictor variables) that correlate with the concentrations of renal markers in serum and urine or with the $\mathrm{Ccr}$ measurements (dependent variables). The $\mathrm{Pb}-\mathrm{B}, \mathrm{Pb}-\mathrm{U}, \mathrm{Pb}-\mathrm{T}$, and $\mathrm{ZPP}-\mathrm{B}$ were entered separately in the model together with potential covariates such as age, the concentration of $\mathrm{Cd}-\mathrm{U}$ and serum $\gamma-\mathrm{GT}$ (as an index of alcohol intake), the presence of hypertension (absence 0; presence 1), smoking habits (never smokers 0; others 1), cohort code (control 0; exposed 1), and the first order interaction terms between the presence of hypertension and the concentrations of $\mathrm{Cd}-\mathrm{U}$ or the four $\mathrm{Pb}$ exposure variables. Significant predictors and covariates were traced by a stepwise regression procedure that ended when all the regression coefficients in the model were significant at the $5 \%$ level of probability. To avoid collinearities between age and other independent variables, age was centered for 45 years. The level of $p=0.05$ was considered as statistically significant.

\section{Results}

CHARACTERISTICS OF THE WORKERS

Table 1 summarises the general characteristics of the different groups. Control and $\mathrm{Pb}$ workers had similar smoking habits: $26 \%$ and $24 \%$ respectively were never smokers, the others were either current or ex-smokers. At the time of the study the total $\mathrm{Pb}$ group showed $\mathrm{Pb}-\mathrm{B}$ and $\mathrm{Pb}-\mathrm{U}$ that were on average three to five times higher than those measured in the total control group. The moderate but significant increases of ZPP-B and $\delta$-ALA in urine reflect an interference of $\mathrm{Pb}$ with the haem-biosynthetic pathway of the $\mathrm{Pb}$ workers. The body burden of $\mathrm{Pb}$ estimated by the measurement of $\mathrm{Pb}-\mathrm{T}$ was on average also about three times higher in the $\mathrm{Pb}$ workers than in their controls (table 1). The striking difference between both groups is illustrated by the cumulative frequency distributions of $\mathrm{Pb}-\mathrm{T}$ (fig 1). The values for $\mathrm{Pb}-\mathrm{T}$ found for the 
Table 1 General characteristics of control and $\mathrm{Pb}$ exposed workers

\begin{tabular}{|c|c|c|c|c|c|c|}
\hline & \multicolumn{3}{|l|}{ Controls } & \multicolumn{3}{|l|}{ Po Exposed } \\
\hline & $\begin{array}{l}\text { Normotensive } \\
(n=55) \oint \|\end{array}$ & $\begin{array}{l}\text { Hypertensive } \\
(n=13) \oint \|\end{array}$ & $\begin{array}{l}\text { Total } \\
(n=68) \Phi \|\end{array}$ & $\begin{array}{l}\text { Normotensive } \\
(n=47) \|\end{array}$ & $\begin{array}{c}\text { Hypertensive } \\
(n=29) \|\end{array}$ & $\begin{array}{l}\text { Total } \\
(n=76) \|\end{array}$ \\
\hline \multirow{2}{*}{$\begin{array}{l}\text { Age }(y) \dagger \\
\text { Years of } \mathrm{Pb} \text { exposure } \dagger\end{array}$} & $43 \cdot 0(9 \cdot 1)$ & $45 \cdot 1(8 \cdot 7)$ & $43.4(9.0)$ & \multirow{2}{*}{$\begin{array}{c}42 \cdot 3(8 \cdot 1) \\
(29 \cdot 4-55 \cdot 8) \\
15 \cdot 9(6 \cdot 8) \\
(5 \cdot 9-35 \cdot 9)\end{array}$} & \multirow{2}{*}{$\begin{array}{c}45 \cdot 7(6 \cdot 8) \\
(29 \cdot 4-55 \cdot 3) \\
20 \cdot 8(7 \cdot 3) \\
(8 \cdot 9-36 \cdot 3)\end{array}$} & \multirow{2}{*}{$\begin{array}{c}43.6(7 \cdot 8) \\
(29 \cdot 4-55 \cdot 8) \\
17 \cdot 7(7 \cdot 3) \\
(5 \cdot 9-36 \cdot 3)\end{array}$} \\
\hline & - & - & - & & & \\
\hline Years of employment $†$ & \multirow{4}{*}{$\begin{array}{c}20.8(9.1) \\
(5.9-40.8) \\
175.5(6.1) \\
(165.0-195.0) \\
76(11) \\
(54-104) \\
1.91(0.14) \\
(1.62-2.31)\end{array}$} & \multirow{4}{*}{$\begin{array}{c}20 \cdot 2(7 \cdot 1) \\
(6 \cdot 9-31 \cdot 5) \\
(175 \cdot 3(6 \cdot 8) \\
(164 \cdot 0-186 \cdot 0) \\
92(14) \\
(74-118) \\
2 \cdot 07(0 \cdot 15) \\
(1 \cdot 90-2 \cdot 37)\end{array}$} & $\begin{array}{l}20.7(8.7) \\
(5.9-40.8)\end{array}$ & - & - & 一 \\
\hline Height $(\mathrm{cm}) \dagger$ & & & $\begin{array}{l}175.5(6 \cdot 2) \\
(164.0-195.0)\end{array}$ & $\begin{array}{c}175 \cdot 0(5 \cdot 3) \\
(163 \cdot 5-188 \cdot 5)\end{array}$ & $\begin{array}{c}174 \cdot 4(6 \cdot 2) \\
(163 \cdot 0-188 \cdot 0)\end{array}$ & $\begin{array}{c}174.8(5 \cdot 6) \\
(163.0-188 \cdot 5)\end{array}$ \\
\hline Weight (kg) $†$ & & & $\begin{array}{l}79(13) \\
(54-118)\end{array}$ & $\begin{array}{l}76 \cdot 5(12) \\
(55-100)\end{array}$ & $\begin{array}{l}84(12) \\
(66-113)\end{array}$ & $\begin{array}{l}79(12) \\
(55-113)\end{array}$ \\
\hline Body surface area $\left(\mathrm{m}^{2}\right) \dagger$ & & & $\begin{array}{c}1.95(0.16) \\
(1.62-2.37)\end{array}$ & $\begin{array}{l}1.91(0.15) \\
(1.63-2.26)\end{array}$ & $\begin{array}{c}1.98(0.15) \\
(1.74-2.32)\end{array}$ & $\begin{array}{c}1.94(0.15) \\
(1.63-2.32)\end{array}$ \\
\hline \multirow{2}{*}{$\begin{array}{l}\text { Blood variables: } ¥ \\
\text { Packed cell volume (\%) }\end{array}$} & \multirow{8}{*}{$\begin{array}{c}46 \cdot 0 \\
(40 \cdot 2-52 \cdot 2) \\
139 \\
(63-261) \\
0 \cdot 6 \\
(0 \cdot 2-2 \cdot 1) \\
1 \cdot 1 \\
(0 \cdot 7-2 \cdot 1) \\
23 \\
(8-135)\end{array}$} & \multirow{8}{*}{$\begin{array}{c}45 \cdot 7 \\
(41 \cdot 5-50 \cdot 8) \\
148 \\
(77-279) \\
0 \cdot 5 \\
(0 \cdot 1-2 \cdot 6) \\
1 \cdot 1 \\
(0 \cdot 8-1 \cdot 4) \\
39 \\
(14-305)\end{array}$} & & \multirow{8}{*}{$\begin{array}{c}45 \cdot 7 \\
(41 \cdot 2-51 \cdot 3) \\
466^{\star \star \star} \\
(342-679) \\
1 \cdot 4^{\star \star \star} \\
(0 \cdot 4-5 \cdot 9) \\
3 \cdot 3^{\star \star \star} \\
(1 \cdot 2-11 \cdot 9) \\
27 \\
(9-352)\end{array}$} & \multirow{8}{*}{$\begin{array}{c}45 \cdot 7 \\
(40 \cdot 8-50 \cdot 8) \\
378^{\star \star \star} \\
(259-566) \\
1 \cdot 0^{\star \star} \\
(0 \cdot 3-3 \cdot 3) \\
2 \cdot 5^{\star \star \star} \\
(1 \cdot 0-17 \cdot 9) \\
34 \\
(13-87)\end{array}$} & \multirow{8}{*}{$\begin{array}{c}45 \cdot 7 \\
(40 \cdot 8-51 \cdot 3) \\
430^{\star \star \star} \\
(259-679) \\
1 \cdot 2^{\star \star \star} \\
(0 \cdot 3-5 \cdot 9) \\
3 \cdot 0^{\star \star \star} \\
(1 \cdot 0-17 \cdot 9) \\
29 \\
(9-352)\end{array}$} \\
\hline & & & $\begin{array}{l}45 \cdot 9 \\
(40 \cdot 2-52 \cdot 2)\end{array}$ & & & \\
\hline $\mathrm{Pb}(\mu \mathrm{g} / \mathrm{l})$ & & & 141 & & & \\
\hline $\mathrm{Cd}(\mu \mathrm{g} / \mathrm{l})$ & & & $\begin{array}{c}(03-219) \\
0.6\end{array}$ & & & \\
\hline & & & $(0 \cdot 1-2 \cdot 6)$ & & & \\
\hline $\begin{array}{l}\text { ZPP } \\
(\mu \mathrm{g} / \mathrm{g} \text { haemoglobin })\end{array}$ & & & $\begin{array}{l}1 \cdot 1 \\
(0 \cdot 7-2 \cdot 1)\end{array}$ & & & \\
\hline Serum $\gamma$ GT & & & 25 & & & \\
\hline (IU/l) & & & $(8-305)$ & & & \\
\hline \multirow{2}{*}{$\begin{array}{l}\text { Urine variables: } \ddagger \\
\mathrm{Pb}(\mu \mathrm{g} / \mathrm{g} \text { creatinine })\end{array}$} & \multirow{7}{*}{$\begin{array}{c}7 \cdot 2 \\
(2 \cdot 8-26 \cdot 2) \\
0 \cdot 52 \\
(0 \cdot 16-1 \cdot 23) \\
3 \cdot 0 \\
(1 \cdot 5-5 \cdot 3) \\
21 \cdot 7 \\
(<15 \cdot 2-69 \cdot 3)\end{array}$} & \multirow{7}{*}{$\begin{array}{c}9 \cdot 0 \\
(4 \cdot 1-18 \cdot 4) \\
0 \cdot 57 \\
(0 \cdot 19-1 \cdot 51) \\
2 \cdot 8 \\
(2 \cdot 1-4 \cdot 6) \\
20 \cdot 2 \\
(<15 \cdot 2-52 \cdot 9)\end{array}$} & & \multirow{7}{*}{$\begin{array}{c}43 \cdot 9^{\star \star \star} \\
(10 \cdot 0-113 \cdot 5) \\
1 \cdot 10^{\star \star \star} \\
(0 \cdot 29-2 \cdot 54) \\
4 \cdot 8^{\star \star \star} \\
(2 \cdot 1-19 \cdot 4) \\
64 \cdot 0^{\star \star \star} \\
(19 \cdot 6-167 \cdot 1)\end{array}$} & \multirow{7}{*}{$\begin{array}{c}33 \cdot 5^{\star \star \star} \\
(15 \cdot 3-104 \cdot 5) \\
0 \cdot 95^{\star \star} \\
(0 \cdot 37-1 \cdot 98) \\
3 \cdot 6^{\star} \\
(2 \cdot 0-7 \cdot 8) \\
69 \cdot 0^{\star \star \star} \\
(21 \cdot 7-162 \cdot 3)\end{array}$} & \multirow{7}{*}{$\begin{array}{l}39 \cdot 6^{\star \star \star} \\
(10 \cdot 0-113 \cdot 5) \\
1 \cdot 04^{\star \star \star} \\
(0 \cdot 29-2 \cdot 54) \\
4 \cdot 3^{\star \star \star} \\
(2 \cdot 0-19 \cdot 4) \\
65 \cdot 8^{\star \star \star} \\
(19 \cdot 6-167 \cdot 1)\end{array}$} \\
\hline & & & $\begin{array}{l}7 \cdot 5 \\
(2 \cdot 8-26 \cdot 2)\end{array}$ & & & \\
\hline $\mathrm{Cd}(\mu \mathrm{g} / \mathrm{g}$ creatinine $)$ & & & 0.53 & & & \\
\hline & & & $(0 \cdot 16-1 \cdot 51)$ & & & \\
\hline $\begin{array}{l}\delta \text {-ALA } \\
\text { (mg/g creatinine) }\end{array}$ & & & $\begin{array}{l}3 \cdot 0 \\
(1 \cdot 5-5 \cdot 3)\end{array}$ & & & \\
\hline $\mathrm{Pb}-\mathrm{T}$ & & & 21.4 & & & \\
\hline$(\mu \mathrm{g} / \mathrm{g}$ bone mineral $) \ddagger$ & & & (1) & & & \\
\hline
\end{tabular}

${ }^{\star} \mathrm{p}<0.05 ;{ }^{\star \star} \mathrm{p}<0.01 ;{ }^{\star \star \star} \mathrm{p}<0.001 v$ respective control group (Student's $t$ test).

tArithmetic mean (SD) (range) and łgeometric mean (range) are shown

SSerum $\gamma$-GT: normotensive $n=42$, hypertensive $n=10$, total $n=52$.

$\| \mathrm{Pb}-\mathrm{T}$ : controls (normotensive $\mathrm{n}=49$, hypertensive $\mathrm{n}=12$, total total $\mathrm{n}=61$ ); $\mathrm{Pb}$ exposed (normotensive $\mathrm{n}=44$, hypertensive $\mathrm{n}=26$, total $\mathrm{n}=70$ ).

exposed group were generally higher than those encountered in other cross sectional surveys of industrially exposed populations. ${ }^{26}$ As a result, this population is well suited to the investigation of the effects of $\mathrm{Pb}$ on renal function.

Biological indices of exposure to $\mathrm{Hg}$ or $\mathrm{Cd}$ indicated that none of the workers (either control or $\mathrm{Pb}$ exposed) was ever excessively exposed to these metals. For $\mathrm{Cd}$, a slightly higher exposure to this metal was found in the

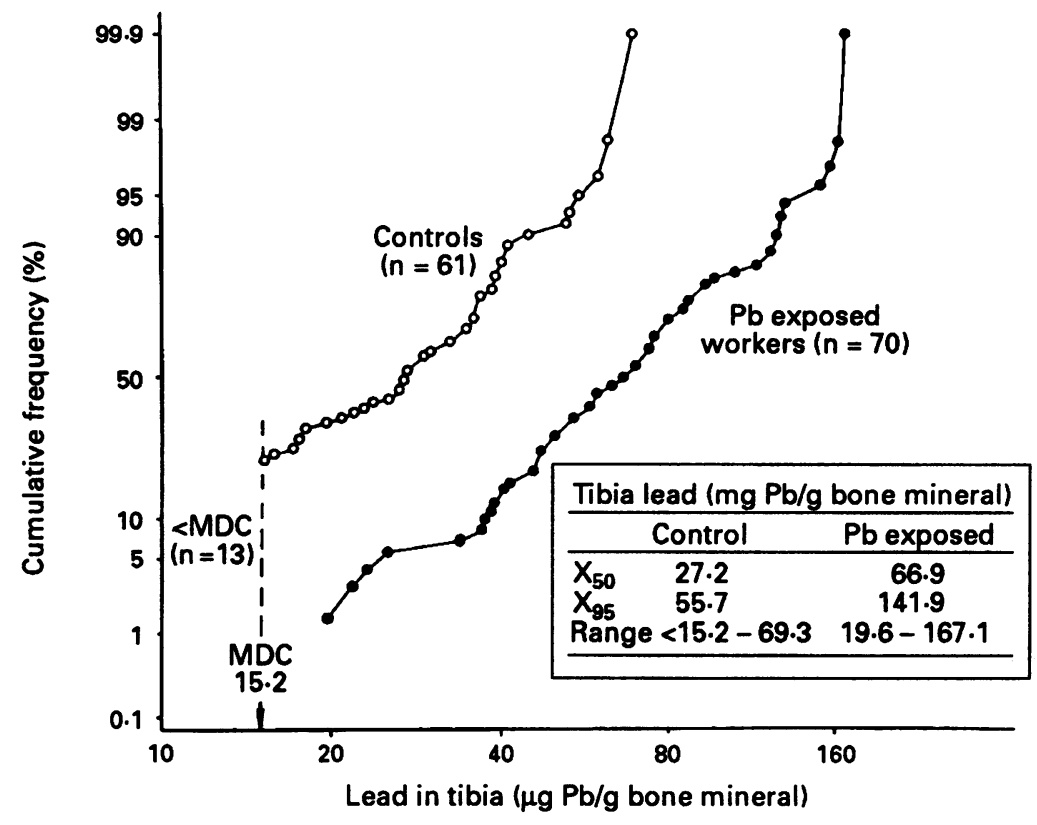

Figure 1 Cumulative frequency distributions of $\mathrm{Pb}$ in tibia $(\mathrm{Pb}-\mathrm{T})$ of control and $\mathrm{Pb}$ exposed workers. Thirteen control workers had Pb-T concentrations below the minimal detectable concentration (MDC) of $15.2 \mu \mathrm{g} \mathrm{Pb/g}$ bone mineral.
$\mathrm{Pb}$ workers as reflected by their concentrations of $\mathrm{Cd}-\mathrm{U}$ and $\mathrm{Cd}-\mathrm{B}$. Their values were, however, within the limits of distributions usually found for groups from the population at large in Belgium. $\mathrm{Hg}-\mathrm{U}$ never exceeded $\mathbf{1 0}$ $\mu \mathrm{g} \mathrm{Hg} / \mathrm{g}$ creatinine.

MARKERS OF NEPHROTOXICITY

The status of renal function was evaluated just before the protein induced hyperfiltration protocol started. Except for NAG-U the mean results for the nine other renal markers did not differ significantly between the exposed and control workers (table 2). Fasting serum concentrations of creatinine and $\beta_{2}$-microglobulin were normal $(<13 \mathrm{mg} / 1$ or $<2 \mathrm{mg} / 1$ respectively) in all subjects; the serum concentrations of urea and uric acid were also normal $(<481 \mathrm{mg} / \mathrm{l}$ and $<75 \mathrm{mg} / 1$, respectively) apart from a few marginally increased results. No significant difference was found between the four subgroups for serum urea, but serum uric acid tended to be somewhat higher in the hypertensive subgroups possibly due to the combined effect of diuretic consumption and a reduced renal excretion of uric acid in hypertension. ${ }^{27}$ The prevalences of increased urinary values of high (albumin, transferrin) and low $\left(\beta_{2}\right.$-microglobulin, RBP) molecular weight proteins did not differ significantly between the $\mathrm{Pb}$ exposed and control group $\left(\chi^{2}, p>0.05\right)$ (table 2$)$. The four subgroups did not differ as to their mean urinary excretion of $\beta_{2}$-microglobulin, $R B P$, and transferrin. Albuminuria in the hypertensive control subgroup was, however, significantly higher than that in the other three subgroups. NAG-U was significantly higher in 
Table 2 Bloodborne and urinary markers of renal function in controls and $\mathrm{Pb}$ exposed workers

\begin{tabular}{|c|c|c|c|c|c|c|}
\hline & \multicolumn{3}{|l|}{ Controls } & \multicolumn{3}{|l|}{ Pb Exposed } \\
\hline & $\begin{array}{l}\text { Normotensive } \\
(n=55) \ddagger\end{array}$ & $\begin{array}{l}\text { Hypertensive } \\
(n=13) \ddagger\end{array}$ & $\begin{array}{l}\text { Total } \\
(n=68) \ddagger\end{array}$ & $\begin{array}{l}\text { Normotensive } \\
(n=47)\end{array}$ & $\begin{array}{l}\text { Hypertensive } \\
(n=29)\end{array}$ & $\begin{array}{l}\text { Total } \\
(n=76)\end{array}$ \\
\hline $\begin{array}{l}\text { Serumt } \\
\beta_{2} \text {-Microglobulin }(\mathrm{mg} / \mathrm{l}) \\
\text { Creatinine }(\mathrm{mg} / \mathrm{l}) \\
\text { Urea }(\mathrm{mg} / \mathrm{l}) \\
\text { Uric acid }(\mathrm{mg} / \mathrm{l})\end{array}$ & $\begin{array}{c}1 \cdot 31 \\
(0 \cdot 92-1 \cdot 95) \\
9 \cdot 7 \\
(7 \cdot 8-12 \cdot 8) \\
324 \\
(233-486) \\
54 \\
(38-81)\end{array}$ & $\begin{array}{l}1 \cdot 23 \\
(1 \cdot 01-1 \cdot 96) \\
9 \cdot 0 \\
(8 \cdot 1-10 \cdot 0) \\
316 \\
(248-414) \\
61 \\
(43-78)\end{array}$ & $\begin{array}{l}1 \cdot 29 \\
(0 \cdot 92-1 \cdot 96) \\
9 \cdot 5 \\
(7 \cdot 8-12 \cdot 8) \\
322 \\
(233-486) \\
55 \\
(38-81)\end{array}$ & $\begin{array}{l}1 \cdot 27 \\
(0 \cdot 97-1 \cdot 86) \\
9 \cdot 1 \\
(6 \cdot 9-10 \cdot 7)^{\star \star} \\
297 \\
(159-503) \\
51 \\
(33-82)\end{array}$ & $\begin{array}{l}1 \cdot 32 \\
(1 \cdot 00-1 \cdot 87) \\
9 \cdot 4 \\
(7 \cdot 3-11 \cdot 5) \\
336 \\
(208-464) \\
57 \\
(40-90)\end{array}$ & $\begin{array}{l}1 \cdot 29 \\
(0 \cdot 97-1 \cdot 87) \\
9 \cdot 2 \\
(6 \cdot 9-11 \cdot 5) \\
311 \\
(159-503) \\
53 \\
(33-90)\end{array}$ \\
\hline $\begin{array}{l}\text { Urine† } \\
\text { Albumin } \\
\text { (mg/g creatinine) } \\
\text { Transferrin } \\
\text { ( } \mu \text { g/g creatinine) } \\
\beta_{2}-\text { Microglobulin } \\
\text { ( } \mu \text { g/g creatinine) } \\
\text { RBP } \\
\text { ( } \mu \text { g/g creatinine) } \\
\text { NAG } \\
\text { (IU/g creatinine) } \\
\text { Kallikrein } \\
\text { (U/g creatinine) }\end{array}$ & $\begin{array}{c}4.9 \\
(0 \cdot 9-21 \cdot 3) \\
451 \\
(137-3768) \\
74 \\
(9-342) \\
66 \\
(16-185) \\
1.07 \\
(0.30-2 \cdot 84) \\
0.58 \\
(0.12-1 \cdot 83)\end{array}$ & $\begin{array}{l}9 \cdot 2 \\
(2 \cdot 0-350 \cdot 4) \\
648 \\
(65-25080) \\
87 \\
(20-840) \\
83 \\
(42-376) \\
1 \cdot 15 \\
(0 \cdot 37-3 \cdot 16) \\
0 \cdot 49 \\
(0 \cdot 11-1 \cdot 16)\end{array}$ & $\begin{array}{l}5 \cdot 6 \\
(0.9-350 \cdot 4) \oint \\
483 \\
(65-25080) \| \\
77 \\
(9-840) \rrbracket \\
69 \\
(16-376)+t \\
1.09 \\
(0 \cdot 30-3 \cdot 16) \\
0.56 \\
(0.11-1.83)\end{array}$ & $\begin{array}{l}4 \cdot 8 \\
(1 \cdot 5-33 \cdot 0) \\
405 \\
(35-7978) \\
62 \\
(10-195) \\
71 \\
(13-354) \\
1.38 \\
(0.57-4.91)^{\star \star} \\
0.53 \\
(0.12-1.96)\end{array}$ & $\begin{array}{c}5 \cdot 8 \\
(1 \cdot 4-25 \cdot 4) \\
472 \\
(38-3093) \\
75 \\
(6-192) \\
63 \\
(21-132) \\
1 \cdot 16 \\
(0 \cdot 6-3 \cdot 47) \\
0 \cdot 60 \\
(0 \cdot 15-2 \cdot 72)\end{array}$ & $\begin{array}{c}5 \cdot 2 \\
(1 \cdot 4-33 \cdot 0) \oint \\
429 \\
(35-7978) \| \\
67 \\
(6-195) \rrbracket \\
68 \\
(13-354)+\dagger \\
1 \cdot 29 \\
(0.57-4.91)^{\star} \\
0.55 \\
(0.12-2 \cdot 72)\end{array}$ \\
\hline
\end{tabular}

${ }^{\star} \mathrm{p}<0.05 ;{ }^{\star \star} \mathrm{p}<0.01 v$ the respective control group (Student's $t$ test).

$t$ Geometric mean (range) is shown.

łUrea in serum and uric acid in serum: normotensive $n=42$, hypertensive $n=10$, total $n=52$.

Control/exposed: $7 / 6$ values $>15 \mathrm{mg} / \mathrm{g}$ creatinine.

$\|$ C/E: $5 / 7$ values $>1750 \mu \mathrm{g} / \mathrm{g}$ creatinine.

IC/E: $2 / 0$ values $>300 \mu \mathrm{g} / \mathrm{g}$ creatinine.

HCC/E: $1 / 1$ values $>300 \mu \mathrm{g} / \mathrm{g}$ creatinine.

the normotensive $\mathrm{Pb}$ subgroup and in the total $\mathrm{Pb}$ group, whereas the prevalence of increased NAG-U values $(>2 \cdot 20 \mathrm{IU} / \mathrm{g}$ creatinine) was not significantly different between the control (4/68) and exposed groups $(11 / 76)$. Neither the activity of urinary kallikrein (an enzymic marker of the distal tubule) nor the prevalence of abnormally low values of activity were different between the groups. The results did not change when two control and three $\mathrm{Pb}$ exposed workers with low natriuria ( $<35 \mathrm{mmol} / \mathrm{g}$ creatinine) were ignored (low sodium intake may cause a pronounced increase of this enzyme).

Stepwise multiple regression analysis on merged subcohorts (controls + exposed) showed that none of these 10 renal markers was related to any $\mathrm{Pb}$ exposure variable and that Cd-U was the only predictor variable that

Table 3 Creatinine clearance rates in control and $\mathrm{Pb}$ exposed workers: measurements under baseline conditions and after an acute oral protein load ( $400 \mathrm{~g}$ cooked meat)

\begin{tabular}{|c|c|c|c|c|}
\hline \multirow{4}{*}{$\begin{array}{l}\text { Normotensive } \\
\text { Baseline } \\
\text { Protein load: } \\
\text { 1st period } \\
\text { 2nd period } \\
\text { Peak period }\end{array}$} & \multicolumn{4}{|c|}{ Creatinine clearance $\ddagger\left(\mathrm{ml} / \mathrm{min} / 1 \cdot 73 \mathrm{~m}^{2}\right)$} \\
\hline & \multicolumn{2}{|l|}{ Controls } & \multicolumn{2}{|l|}{ Pb exposed } \\
\hline & $\begin{array}{l}n=55 \\
114 \cdot 2\end{array}$ & $(81-156)$ & $\begin{array}{l}n=47 \\
123 \cdot 5^{\star \star}\end{array}$ & $(97-177)$ \\
\hline & $\begin{array}{l}126 \cdot 0 \\
125 \cdot 4 \\
130 \cdot 2 \dagger\end{array}$ & $\begin{array}{l}(101-191) \\
(101-194) \\
(105-194)\end{array}$ & $\begin{array}{l}136 \cdot 8^{\star \star} \\
135 \cdot 6^{\star \star} \\
143 \cdot 0 十^{\star \star \star}\end{array}$ & $\begin{array}{r}(102-182) \\
(99-198) \\
(111-198)\end{array}$ \\
\hline $\begin{array}{l}\text { Hypertensive } \\
\text { Baseline } \\
\text { Protein load: }\end{array}$ & $\begin{array}{l}n=13 \\
121 \cdot 2\end{array}$ & $(106-156)$ & $\begin{array}{l}n=29 \\
117 \cdot 8\end{array}$ & $(88-155)$ \\
\hline $\begin{array}{l}\text { 1st period } \\
\text { 2nd period } \\
\text { Peak period }\end{array}$ & $\begin{array}{l}135 \cdot 1 \\
131 \cdot 0 \\
137 \cdot 5 \dagger\end{array}$ & $\begin{array}{l}(112-175) \\
(110-159) \\
(120-175)\end{array}$ & $\begin{array}{l}131 \cdot 9 \\
127 \cdot 2 \\
136 \cdot 8 \dagger\end{array}$ & $\begin{array}{l}(96-189) \\
(95-157) \\
(96-189)\end{array}$ \\
\hline $\begin{array}{l}\text { Total } \\
\text { Baseline } \\
\text { Protein load: }\end{array}$ & $\begin{array}{l}n=68 \\
115.5\end{array}$ & $(81-156)$ & $\begin{array}{l}n=76 \\
121 \cdot 3^{\star}\end{array}$ & $(88-177)$ \\
\hline $\begin{array}{l}\text { 1st period } \\
\text { 2nd period } \\
\text { Peak period }\end{array}$ & $\begin{array}{l}127 \cdot 7 \\
126 \cdot 5 \\
131 \cdot 6 \dagger\end{array}$ & $\begin{array}{l}(101-191) \\
(101-194) \\
(105-194)\end{array}$ & $\begin{array}{l}134 \cdot 9^{\star} \\
132 \cdot 3^{\star} \\
140 \cdot 6 \dagger^{\star \star}\end{array}$ & $\begin{array}{l}(96-189) \\
(95-198) \\
(96-198)\end{array}$ \\
\hline
\end{tabular}

${ }^{\star} \mathrm{p}<0.05 ;{ }^{\star \star} \mathrm{p}<0.01 ;{ }^{\star \star \star} \mathrm{p}<0.001 v$ the control group (Student's $t$ test).

tp $<0.0001 v$ baseline (paired $t$ test: $\log$ PCcr - log BCcr - that is, log PCcr/BCcr).

$\neq$ Geometric mean (range) is shown. significantly correlated with NAG-U ( $\mathrm{n}=$ 128, $r=0.44, \mathrm{p}<0.0001)$. Cohort code did not emerge as significant covariate of NAG$\mathrm{U}$, indicating that NAG-U was only associated with the renal burden of $\mathrm{Cd}$ (reflected as Cd-U) irrespective of whether they were controls or $\mathrm{Pb}$ workers. Similar correlation characteristics between NAG-U and Cd-U were found for the control group ( $\mathrm{n}=68, r=0.41$, slope $=0.331, \mathrm{p}<0.005)$ and the $\mathrm{Pb}$ exposed group $(\mathrm{n}=76, \quad r=0.35, \quad$ slope $=0.401$; $\mathrm{p}<0.001)$.

\section{CREATININE CLEARANCE AND PROTEIN} INDUCED HYPERFILTRATION

Table 3 summarises the results of the protein induced hyperfiltration measurements. During the whole examination period the diuresis was maintained at about $6-7 \mathrm{ml} / \mathrm{min}$ on average and was not significantly different between controls and $\mathrm{Pb}$ exposed subjects (results not shown). In each subject the serum creatinine concentration slightly increased after the oral protein load. At PCcr it amounted on average to 10.4 and $10.1 \mathrm{mg} / \mathrm{l}$ in the control and $\mathrm{Pb}$ workers respectively. All the subjects had a normal BCcr (all values $>80 \mathrm{ml} / \mathrm{min} / 1 \cdot 73 \mathrm{~m}^{2}$ ). On a group basis (total), the $\mathrm{Pb}$ workers had a mean BCcr slightly higher than that of their controls $\left(121.3 v 115.5 \mathrm{ml} / \mathrm{min} / 1.73 \mathrm{~m}^{2}\right.$; $\mathrm{p}=0.04$ ) and their mean PCcr was 9 $\mathrm{ml} / \mathrm{min} / 1.73 \mathrm{~m}^{2}$ higher $(140.6 v 131.6 ; \mathrm{p}=$ $0.004)$. Likewise, the normotensive $\mathrm{Pb}$ workers showed significantly higher BCcr and PCcr than their controls, whereas in the hypertensive workers no difference was found between the control and $\mathrm{Pb}$ exposed subgroups. Whatever the group of control or $\mathrm{Pb}$ exposed workers, the paired $t$ test (on log values) between $\log \mathrm{PCcr}$ and $\log \mathrm{BCcr}$ invariably showed a highly significant $(\mathrm{p}<$ 0.0001 ) relative increase in Ccr after oral 
protein load. Neither this relative increase (on average from 13 to $16 \%$ ) nor the haemodynamic response (on average from 16.0 to 19.6 $\mathrm{ml} / \mathrm{min} / 1.73 \mathrm{~m}^{2}$ ) after protein load were significantly different, however, when comparing the control workers (normotensive, hypertensive, total) with the corresponding $\mathrm{Pb}$ exposed workers (table 4).

Table 4 Haemodynamic response and relative increase of creatinine clearance after an acute oral protein load ( $400 \mathrm{~g}$ cooked meat) in control and $\mathrm{Pb}$ exposed workers

\begin{tabular}{|c|c|c|c|c|}
\hline & Controls & & $P b$ exposed & \\
\hline $\begin{array}{l}\text { Normotensive } \\
\Delta \mathrm{Ccr}\left(\mathrm{ml} / \mathrm{min} / 1 \cdot 73 \mathrm{~m}^{2}\right)^{\star} \\
\text { Ratio PCcr/BCcrf }\end{array}$ & $\begin{array}{l}\mathrm{n}=55 \\
16 \cdot 0(11 \cdot 3) \\
1 \cdot 14\end{array}$ & $\begin{array}{l}(-8 \text { to } 50) \\
(0.95-1 \cdot 62)\end{array}$ & $\begin{array}{l}\mathrm{n}=47 \\
19 \cdot 6(15 \cdot 9) \ddagger \\
1.16 \ddagger\end{array}$ & $\begin{array}{l}(-1 \text { to } 78) \\
(0.99-1 \cdot 75)\end{array}$ \\
\hline $\begin{array}{l}\text { Hypertensive } \\
\Delta \mathrm{Ccr}\left(\mathrm{ml} / \mathrm{min} / 1 \cdot 73 \mathrm{~m}^{2}\right) \\
\text { Ratio } \mathrm{PCcr} / \mathrm{BCcr}\end{array}$ & $\begin{array}{l}\mathrm{n}=13 \\
16 \cdot 3(10 \cdot 9) \\
1 \cdot 13\end{array}$ & $\begin{array}{l}(-5 \text { to } 31) \\
(0 \cdot 96-1 \cdot 25)\end{array}$ & $\begin{array}{l}n=29 \\
19 \cdot 1(16 \cdot 4) \ddagger \\
1 \cdot 16 \ddagger\end{array}$ & $\begin{array}{l}(-9 \text { to } 83) \\
(0.93-1 \cdot 78)\end{array}$ \\
\hline $\begin{array}{l}\text { Total } \\
\Delta \mathrm{Ccr}\left(\mathrm{ml} / \mathrm{min} / 1 \cdot 73 \mathrm{~m}^{2}\right) \\
\text { Ratio } \mathrm{PCcr} / \mathrm{BCcr}\end{array}$ & $\begin{array}{l}n=68 \\
16 \cdot 1(11 \cdot 2) \\
1 \cdot 14\end{array}$ & $\begin{array}{l}(-8 \text { to } 50) \\
(0.95-1.62)\end{array}$ & $\begin{array}{l}\mathrm{n}=76 \\
19 \cdot 4(16 \cdot 0) \ddagger \\
1 \cdot 16 \ddagger\end{array}$ & $\begin{array}{l}(-9 \text { to } 83) \\
(0.93-1.78)\end{array}$ \\
\hline
\end{tabular}

${ }^{\star}$ Haemodynamic response $=$ difference between peak creatinine clearance after oral protein load and baseline creatinine clearance; arithmetic mean (SD) (range) is shown.

tRatio between peak creatinine clearance (PCr) and baseline creatinine clearance (BCcr); geotRatio between peak creatinine

$\ddagger$ Not significantly different from the control group (Student's $t$ test).

Figure 2 Age related decline of baseline creatinine clearance and peak creatinine clearance after acute protein load in control and $\mathrm{Pb}$ exposed workers (y axis: log scale).

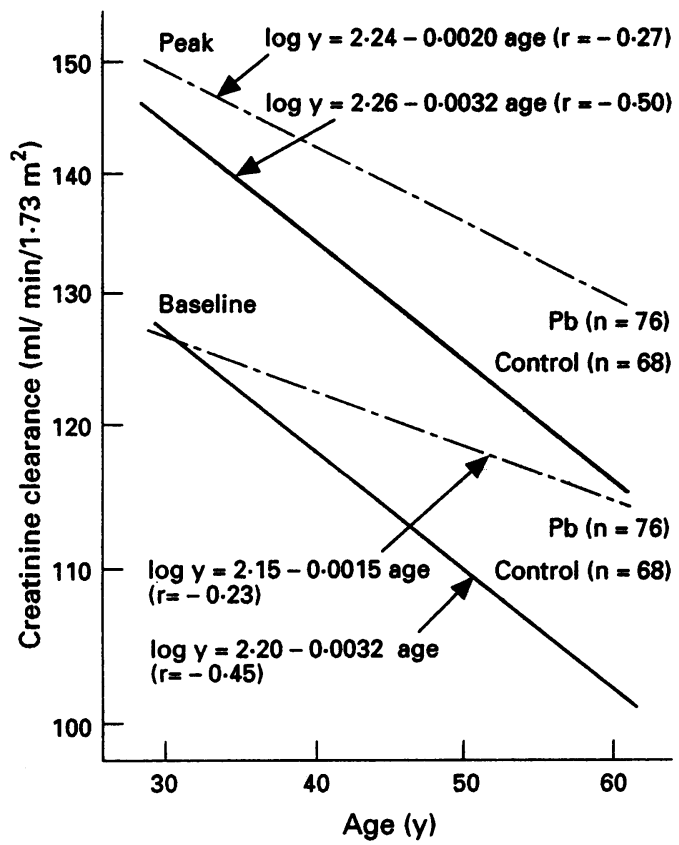

Stepwise multiple regression analysis was performed on merged subcohorts (controls and exposed, distinguished by the categorical variable "cohort code") to identify significant determinants of the BCcr or the PCcr after an oral protein load (table 5 ). $\mathrm{Pb}$ exposure variables (Pb-B, Pb-T, Pb-U, or ZPP-B) were considered separately in the model. Whatever the model, age was, as expected, negatively associated with both Ccr measurements, whereas the haemodynamic response and the ratio $\mathrm{PCcr} / \mathrm{BCcr}$ were independent of age (results not shown).

The close age matching of the controls and lead workers allowed a sound and direct comparison between both subcohorts for the effect of age on BCcr or PCcr. Simple linear regression analysis showed significant inverse relation between age and $\mathrm{BCcr}$ (control: $r=$ $-0.45, \mathrm{p}<0.001 ; \mathrm{Pb}: r=-0.23, \mathrm{p}=0.05)$ or PCcr (control: $r=-0.50, \mathrm{p}<0.001$; $\mathrm{Pb}$ : $r=-0.27, \mathrm{p}<0.05$ ) (fig 2). These age related declines were less pronounced in the $\mathrm{Pb}$ subcohort compared with the controls; however, the slope was significantly less negative in the $\mathrm{Pb}$ workers only for BCcr ( $t$ test on slope, $p<0.05)$. Their age related decrease in BCcr over a lifespan of 30 years (from 30 to 60 years of age) was on average half that found in the control workers $(0.41 v 0.84$ $\mathrm{ml} / \mathrm{min} / 1 \cdot 73 \mathrm{~m}^{2}$ per year).

With regard to the $\mathrm{Pb}$ exposure variables the stepwise multiple regression analysis showed the same results with $\mathrm{Pb}-\mathrm{B}, \mathrm{Pb}-\mathrm{U}$, and ZPP-B - that is, none of these variables emerged as a predictor of BCcr or PCcr; however, the results were different when $\mathrm{Pb}-\mathrm{T}$ was introduced as $\mathrm{Pb}$ exposure variable. This showed a modest but positive and statistically significant association with $\mathrm{BCcr}$ or PCcr. Depending on the biological exposure variables ( $\mathrm{Pb}-\mathrm{B}$ or $\mathrm{Pb}-\mathrm{T}$ ), $\mathrm{Cd}-\mathrm{U}$ or serum $\gamma$-GT was also positively associated with PCcr. Cohort code did not emerge as a significant covariate of $\mathrm{BCcr}$ or PCcr, indicating that the association with $\mathrm{Pb}-\mathrm{T}$ or the lack of association with $\mathrm{Pb}-\mathrm{B}, \mathrm{Pb}-\mathrm{U}$, or $\mathrm{ZPP}-\mathrm{B}$ are irrespective of whether they were controls or $\mathrm{Pb}$ workers.

Table 5 Determinants of creatinine clearance rates under baseline conditions and after protein induced hyperfiltration

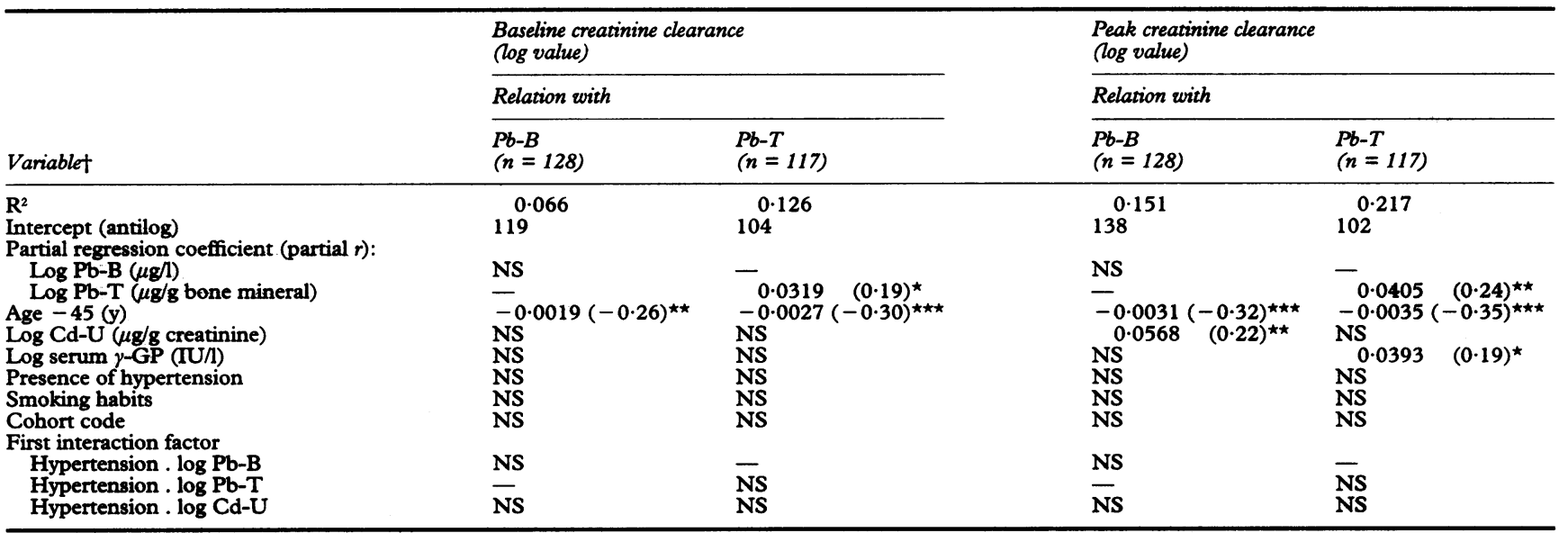

${ }^{\star} \mathrm{p}<0.05 ;{ }^{\star \star} \mathrm{p}<0.01 ;{ }^{\star \star \star} \mathrm{p}<0.001$ for partial $r$.

tDetails on variables: see data processing and statistical analysis. 


\section{Discussion}

The cohort of $\mathrm{Pb}$ workers examined in the present study had a significantly increased internal dose of $\mathrm{Pb}$. Measurements of $\mathrm{Pb}-\mathrm{T}$ suggest that on average their body burden was three times higher than that of the control subjects. Their current $\mathrm{Pb}-\mathrm{B}$ was also about three times higher but did not exceed 700 $\mu \mathrm{g} / \mathrm{l}$. From the historical records of urinary $\delta$ $\mathrm{ALA}$ and $\mathrm{Pb}-\mathrm{B}$ it may also be inferred that $\mathrm{Pb}$ exposure in this smelter only rarely gave rise to $\mathrm{Pb}-\mathrm{B}$ above $700 \mu \mathrm{g} / 1$ during the past 25 years. We have confirmed our previous findings $^{5628}$ and those of other cross sectional studies $^{29-33}$ that in adult male workers with long term moderate occupational exposure to $\mathrm{Pb}$, no significant change in the urinary excretion of low or high molecular weight proteins is detected and that serum concentrations of creatinine, $\beta_{2}$-microglobulin, and uric acid were not affected. It is interesting to note, however, that the $\mathrm{Pb}-\mathrm{T}$ (median) is on average five times higher in the present group of active $\mathrm{Pb}$ smelter workers than in the group studied at a Swedish $\mathrm{Pb}$ smelter. ${ }^{33}$ The increased mean NAG-U found in the $\mathrm{Pb}$ workers is likely caused only by their concomitant slight exposure to $\mathrm{Cd}$ as it was already noted in our previous study. ${ }^{6}$ The increased NAG-U shown by other investigators $^{29} 32$ in $\mathrm{Pb}$ exposed workers should be interpreted with caution as potential, albeit slight, Cd exposure has usually not been considered as a possible covariate.

The examined population was thus suitable to assess whether in asymptomatic workers, an increased $\mathrm{Pb}$ body burden may decrease the renal haemodynamic response to an acute oral protein load. No such effect was detected. This indicates that the changes in urinary prostaglandin secretion detected during our previous study on workers exposed to $\mathrm{Pb}$ apparently has no deleterious consequences for renal haemodynamics. In fact, the most intriguing finding in the present study is that both the BCcr and stimulated Ccr were not only significantly increased in $\mathrm{Pb}$ exposed workers but also positively correlated with the $\mathrm{Pb}$ body burden estimated on the basis of $\mathrm{Pb}-$ $T$. It is unlikely that this finding is fortuitous or due to an uncontrolled bias. Both subgroups of $\mathrm{Pb}$ workers (normotensive and hypertensive) were indeed well matched with their corresponding controls for all factors that may exert an influence on renal function and the study was carried out with a protocol that guaranteed exactly the same procedures for all workers. Also, multiple regression analysis on the separate subcohorts supports the contention that the relations between the creatinine clearance rates and $\mathrm{Pb}-\mathrm{T}$ as shown in table 5 are real and not a product of an artifact produced by merging the control and $\mathrm{Pb}$ subcohorts. The data reported here are not the first to show an increased renal filtration capacity in $\mathrm{Pb}$ workers. Significantly higher Ccr values have been reported in other studies conducted on moderately exposed male $\mathrm{Pb}$ workers $^{2833}$ or on middle aged adults with documented childhood $\mathrm{Pb}$ poisoning. ${ }^{34} \mathrm{~A}$ recent experimental study has also shown that in rats given chronically high doses of $\mathrm{Pb}$, the glomerular filtration rate progressively rose until month 9 then decreased from month 12 when tubulointerstitial nephritis was present. ${ }^{35}$

One might also argue that $\mathrm{Pb}-\mathrm{T}$ still represents a potential source of endogenous $\mathrm{Pb}$ exposure due to $\mathrm{Pb}$ release during bone turnover (for example, the age related rate of radial bone loss in midlife men amounts to $0.45 \%$ per year ${ }^{36}$ ). The elimination of $\mathrm{Pb}$ from the skeleton after occupational exposure to $\mathrm{Pb}$, however, seems to occur rather slowly in retired $\mathrm{Pb}$ workers-namely, biological half life of 16 years for finger bone ${ }^{37}$ or calcaneum and 27 years for the tibia. ${ }^{38}$ On the other hand, the study by Wittmers et al ${ }^{39}$ showed that $\mathrm{Pb}$ concentrations in compact and trabecular bone sites increase until 70 years of age and that beyond this age the cortical bone $\mathrm{Pb}$ values seemed to continue to rise, whereas those of trabecular bone declined. In view of this evidence, it seems unlikely that in the absence of other risk factors the age related kinetics of bone $\mathrm{Pb}$ may mobilise a sufficient amount of $\mathrm{Pb}$ to induce a delayed $\mathrm{Pb}$ nephropathy in subjects whose historical occupational exposure can be qualified as moderate. This hypothesis is supported by the finding by Gerhardsson et al ${ }^{33}$ that the BCcr in a group of 30 retired lead workers (on average: age, 68 years; tibia and calcaneum $\mathrm{Pb}, 39$ and $100 \mu \mathrm{g} \mathrm{Pb} / \mathrm{g}$ bone mineral; Pb-B, $100 \mu \mathrm{g}$ $\mathrm{Pb} / \mathrm{l})$ seven years after cessation of $\mathrm{Pb}$ exposure is similar to that of age matched controls whose concentrations of $\mathrm{Pb}-\mathrm{T}, \mathrm{Pb}$ in calcaneum, and $\mathrm{Pb}-\mathrm{B}$ were three times lower.

In conclusion, the present study suggests that, contrary to our expectation based on the previously noted changes in the urinary excretion of eicosanoids, a moderate exposure to $\mathrm{Pb}$ may be associated with a slight hyperfiltration state. This phenomenon significantly attenuates the age related decline of the BCcr in the present $\mathrm{Pb}$ cohort (on average by a factor of two) compared with that in the control cohort showing on average a yearly decrease of $0.8 \mathrm{ml} / \mathrm{min} / 1.73 \mathrm{~m}^{2}$ for the age range from 30 to 60 years (fig 2). It does not seem likely that this difference between control and $\mathrm{Pb}$ workers may be attributable to a potential selection bias operating on the level of the subcohorts examined. Indeed, the preemployment medical criteria and routine health surveillance programmes were the same for both control and $\mathrm{Pb}$ workers. Although the health significance of the $\mathrm{Pb}$ induced hyperfiltration effect is unknown, it can be concluded that the results of this study support our previous suggestion that compliance with the European Commission Directive ${ }^{40}$ on $\mathrm{Pb}$ exposure $(\mathrm{Pb}-\mathrm{B}<700 \mu \mathrm{g} / \mathrm{l})$ prevents the occurrence of adverse renal changes in most adult male workers. As male workers are usually screened for the absence of renal risk factors at pre-employment medical examination, however, this conclusion may not necessarily be extrapolated to the general population, in which other factors might increase the renal susceptibility to $\mathrm{Pb} .{ }^{41}$ 


\section{Appendix}

\section{Conversion of units:}

$\begin{array}{ll}\mathrm{Pb} 1 \mu \mathrm{g} & =4.83 \mathrm{nmol} \\ \mathrm{Cd} 1 \mu \mathrm{g} & =8.90 \mathrm{nmol} \\ \mathrm{Hg} 1 \mu \mathrm{g} & =4.99 \mathrm{nmol}\end{array}$

Creatinine $1 \mathrm{~g}=8.84 \mathrm{mmol}$

This study was supported by the Science Policy Office of the Prime Minister's Service (Brussels), the International Lead Zinc Research Organisation, and the Commission of the European Union. We are grateful to Messrs E Buyle European Union. We are grateful to Messrs E Buyle,
J Casters, W De Vil, X Dumont, and T Seminck for their skilJ Casters, Mearman and J E Palethorpe for their help in undertaking the $\mathrm{Pb}-\mathrm{T}$ measurements.

1 Bennett WM. Lead nephropathy. Kidney Int 1985;28: $212-20$

2 Koster J, Erhardt A, Stoeppler M, Mohl C, Ritz E. Mobilizable lead in patients with chronic renal failure. Eur 7 Clin Invest 1989;19:228-33.

3 Selevan SG, Landrigan PJ, Stern FB, Jones JH. Mortality of lead smelter workers. Am f Epidemiol 1985;112:673-83.

4 Cooper WC. Deaths from chronic renal disease in U.S battery and lead production workers. Environ Health Perspect 1988;78:61-3.

5 Gennart JPh, Bernard A, Lauwerys R. Assessment of thyroid, testes, kidney and autonomic nervous system thyrotion in lead-exposed workers. Int Arch Occup function in lead-exposed wo

6 Cárdenas A, Roels H, Bernard AM, Barbon R, Buchet JP Lauwerys RR, et al. Markers of early renal changes induced by industrial pollutants. II. Application to workers exposed to lead. $\mathrm{Br} \mathcal{F}$ Ind Med 1993;50:28-36.

7 Bosch JP, Saccaggi A, Lauer A, Ronco C, Belledonne M, Glabman S. Renal functional reserve in humans: effect of protein intake on glomerular filtration rate. $A m \mathcal{f} M e d$ 1983;75:943-50.

8 Levine MM, Kirschenbaum MA, Chaudhari A, Wong MW, Bricker NS. Effect of protein on glomerular filtration rate and prostanoid synthesis in normal and uremic rats. Am 7 Physiol 1986;251:F635-41

9 Rosenberg ME, Swanson JE, Thomas BL, Hostetter TH Glomerular and hormonal responses to dietary protein intake in human renal disease. Am F Physiol 1987;253 F1083-90.

10 Vanrenterghem YF, Verberckmoes RK, Roels LM, Michielsen PJ. Role of prostaglandins in protein-induce glomerular hyperfiltration in normal humans. $A m$ Physiol 1988;254:F463-9.

11 Bosch JP, Lauer A, Glabman S. Short-term protein loading in assessment of patients with renal disease. $A m \mathcal{F}$ Med 1984;77:873-9.

12 Roels HA, Lauwerys RR, Bernard AM, Buchet JP, Vos A Oversteyns $M$. Assessment of the filtration reserve capacity of the kidney in workers exposed to cadmium. capacity of the kidney in work

13 Bernard AM, Lauwerys RR. Continous-flow system for automation of latex immunoassay by particle counting. Clin Chem 1983;29:1007-11.

14 Claeys F, Ducoffre G, Sartor F, Roels H. Analytical quality control of cadmium and lead in blood and cadmium in urine: results of its implementation during a five-year epidemiological study. In: Nordberg GF, Herber RFM, Alessio L, eds. Cadmium in the human environment: toxicity and carcinogenicity. Lyon: International Agency for Research on Cancer, 1992:83-92.

15 Zinterhofer IJ, Jatlow PI, Anthony F. Atomic absorption determination of lead in blood and urine in the presence determination of lead in blood and urine in

16 Magos L, Clarkson TW. Atomic absorption determination of total, inorganic and organic mercury in blood. fourmal of the Association of Official Analytical Chemists 1972;55 966-71.

17 Lauwerys R, Buchet JP, Roels H, Bernard A. Le contrôle de qualité des analyses biologiques dans le domaine de la toxicologie industrielle. Cahiers Méd Trav 1987;24:5-9.

18 Roels H, Abdeladim S, Ceulemans E, Lauwerys R Relationships between the concentrations of mercury in air and in blood or urine in workers exposed to mercury vapour. Ann Occup Hyg 1987;31:135-45.

19 Roels H, Abdeladim S, Braun M, Malchaire J, Lauwerys
R. Detection of hand tremor in workers exposed to mercury vapor: a comparative study of three methods. Environ Res 1989;49:152-65.

20 Roels H, Van de Voorde R, Mata Vargas VM, Lauwerys $\mathrm{R}$. Relationship between atmospheric and urinary nickel in workers manufacturing electrical resistances using nickel oxide: role of the bioavailability of nickel. Occup nickel oxide: role of the

21 Lauwerys RR, Delbroeck R, Vens MD. Automated analysis of delta-aminolaevulinic acid in urine. Clin Chim Acta 1972;40:443-7.

22 Tucker SM, Boyd PJR, Thompson AE, Price RG. Automatic assay of $N$-acetyl- $\beta$-D-glucosaminidase in normal and pathological urine. Clin Chim Acta 1975; 62:333-9.

23 Roels HA, Lauwerys RR, Buchet JP, Bernard AM, Lijnen $P$, Van Houte $G$. Urinary kallikrein activity in workers exposed to cadmium, lead or mercury. Br $\mathcal{F}$ Ind Med 1990;47:331-7.

24 Chettle DR, Scott MC, Somervaille LJ. Lead in bone: sampling and quantitation using $\mathrm{K}$ X-rays excited by ${ }^{109} \mathrm{Cd}$. Environ Health Perspect 1991;91:49-55.

25 Green S, Bradley DA, Palethorpe JE, Maerman D, Chettle $\mathrm{DR}$, Lewis $\mathrm{AD}$, et al. An enhanced sensitivity K-shell $x$-ray fluorescence technique for tibial lead determination. Phys Med Biol 1993;38:389-96.

26 Green S, Bradley DA, Roels HA, Mountford PJ, Morgan WD, Chettle DR, et al. Development and calibration of an in vivo bone lead measurement system, and its application to an industrially exposed population. In: Ellis KJ Eastman JD, eds. Human Body composition. New York: Plenum Press, 1993:295-8.

27 Breckenridge A. Hypertension and hyperuricaemia. Lancet 1966;i:15-8.

28 Buchet JP, Roels H, Bernard A, Lauwerys R. Assessment of renal function of workers exposed to inorganic lead cadmium or mercury vapor. $\mathcal{F}$ Occup Med 1980;22 $741-50$

29 Verschoor M, Wibowo A, Herber R, Van Hemmen J Zielhuis R. Influence of occupational low-level lead exposure on renal parameters. Am f Ind Med 1987;12. 341-51.

30 Huang J, He F, Yigun W, Zhang S. Observations on renal function in workers exposed to lead. Sci Total Environ 1988;71:535-7.

31 Omae $K$ Sakurai $H$, Higaschi $T$, Muto $T$, Ichikawa $M$ Sasaki N. No adverse effects of lead on renal function in lead-exposed workers. Ind Health 1990;23:77-83.

32 Meyer BR, Fischbein A, Rosenman K, Lerman Y, Draye $\mathrm{DE}$, Reidenberg MM. Increased urinary enzyme excretion in workers exposed to nephrotoxic chemicals. $A m \mathcal{F}$ Med 1984;76:989-98.

33 Gerhardsson L, Chettle DR, Englyst V, Nordberg GF Nyhlin H, Scott MC, et al. Kidney effects in long term exposed lead smelter workers. $\mathrm{Br}$ f Ind Med 1992;49: 186-92.

$34 \mathrm{Hu} \mathrm{H}$. A 50-year follow-up of childhood plumbism: hypertension, renal function and hemoglobin levels among survivors. Am f Dis Child 1991;45:681-7.

35 Khalil-Manesh F, Gonick HC, Cohen AH, Alinovi R, Bergamaschi E, Mutti A, et al. Experimental model of lead nephropathy. I. Continuous high-dose lead administration. Kidney Int 1992;41:1192-203.

36 Slemendo CW Christian JC, Reed T, Reister TK, Williams CJ, Johnston CC. Long-term bone loss in men effects of genetic and environmental factors. Ann Inter Med 1992;117:286-91.

37 Nilsson U, Attewell R, Christoffersson JO, Schütz A, Ahlgren L, Skerfving S, Mattsson S. Kinetics of lead in bone and blood after end of occupational exposure. Pharmacol Toxicol 1991;68:477-84.

38 Gerhardsson L, Attewell R, Chettle DR, Englyst V, Lundström NG, Nordberg GF, et al. In vivo measurements of lead in bone in long-term exposed lead smelter ments of lead in bone in long-term exposed lead

39 Wittmers LE, Wallgren J, Alich A, Aufderheide AC, Rapp $\mathrm{G}$. Lead in bone. IV. Distribution of lead in the human skeleton. Arch Environ Health 1988;43:381-91.

40 Council Directive of 28 July $1982(82 / 605 /$ EEC) on the protection of workers from the risks related to exposure to metallic lead and its ionic compounds at work (first ind vidual Directive within the measuring of Article 8 of Directive 80/1107/EEC). Official fournal of the European Community 1982;25:12-21. N ${ }^{\circ}$ L247 23.8.82.

41 Staessen JA, Lauwerys RR, Buchet JP, Bulpitt CJ, Rondia $\mathrm{D}$, Vanrenterghem $\mathrm{Y}$, et al. Impairment of renal function with increasing blood lead concentrations in the general population. NEngl f Med 1992;327:151-6. 